\title{
An Investigation on Psychosocial Factors Impacting Frailty in Older Adults: A Case of Singapore
}

\author{
Yuezhong Liu'*1*, Bing Xun Chia ${ }^{2}$, Yong Joo Loh ${ }^{3}$ and Yin-Leng Theng ${ }^{1}$ \\ ${ }^{1}$ Ageing Research Institute for Society and Education (ARISE), Nanyang Technological University, Singapore \\ ${ }^{2}$ Wee Kim Wee School of Communication and Information, Nanyang Technological University, Singapore \\ ${ }^{3}$ Department in Rehabilitation Medicine, Tan Tock Seng Hospital (TTSH), Singapore
}

Submission: November 04, 2021; Published: November 11, 2021

*Corresponding author: Yuezhong Liu, Ageing Research Institute for Society and Education (ARISE), Nanyang Technological University, Singapore, Email: yz.liu@ntu.edu.sg

\begin{abstract}
Frailty is a geriatric syndrome of increased vulnerability due to diminished physiologic reserves. It is still unclear how the psychosocial factors and physical frailty are related to health outcomes. This study presents prevalence, related physical and psychosocial factors of frailty in older adults in Singapore and the mediation effect of frailty among psychosocial factors.
\end{abstract}

\section{Methods}

Using a cross-sectional analysis of 491 individuals over 65 years of age from 17 community centers, frailty conditions were grouped as Robust, Prefrail and Frail. Psychosocial profiles were collected through established Depression, Social Support, Quality of Life and Loneliness scales.

\section{Results}

Approximately $60.4 \%$ of the participants were between 65 and 74 years of age and $70.3 \%$ were women with $3.3 \%$ and $37.1 \%$ of participants rated as frail and pre-frail, respectively. Results indicated that depression, loneliness, and fall efficacy were significantly lower while social support and quality of life higher in the robust participants compared to prefrail and frail participants. Pathway analyses examined the mediation effect of frailty from psychosocial factors (depression, loneliness, social support) to quality of life. Depression had direct significant association with both frailty $(B=0.034, p<.001)$ and quality of life $(B=-0.497, p<.001)$.

\section{Conclusions}

Frailty has been validated as a mediator on the pathway from psychosocial conditions to health. Psychosocial interventions should be considered further to reduce frailty and improve quality of life. Longitudinal research combining both physical and psychosocial factors are promising to explore the pathways to frailty and its adverse health outcomes.

Keywords: Frailty; Psychosocial Factors; Depression; Social Support; Loneliness; Pathway Analysis

\section{Introduction}

Worldwide and in Singapore, the population is ageing. Until now, Singapore's older adults (aged 65 years and above) constitute $15.2 \%$ of the total population [1]. This is expected to be $53.4 \%$ in 2050 [2]. Frailty is a condition in which the individual is in a vulnerable state at increased risk of adverse health outcomes and/ or dying when exposed to a stressor [3]. It is usually recognized in older adults and leads to adverse health effects [4,5]. Frail people have a significantly higher risk of falls, disability, long-term care and even leading to death [6,7]. In a report which reviewed 21 community-based cohort studies of 61,500 elderly people, the prevalence rate of frailty among older adults aged 65 or more is
9.9\% [8]. Global data from more than 120,000 senior citizens of 28 countries suggests a $43.4 \%$ frailty incidence rate [9]. While it is generally believed that frailty increases with age, no consensus has been reached on the prevalence of frailty [8]. Singapore is a global business hub with 4.04 million residents, and the median age of the resident population rise to reach 41.5 years [1]. It is also one of the fastest ageing nations in the world. The old-age support ratio of residents computed as the ratio of residents aged 20-64 years for each resident aged 65 years and over, declined further to $4.3 \%$ until 2020 [1]. One of the most urgent ageing issues to address is frailty [10]. That is, high prevalence among older adults and increased risk of adverse health outcomes such as falls, disability, 
depression, hospitalization, medical costs, and mortality [4,1114]. Singapore's government has responded to the ongoing frailty movement [15]. In the past decade, many studies have examined the association between frailty and socio-demographic factors. However, few studies focus on the psychosocial factors and their impact on frailty prevalence in Singapore [13,15-17]. highlighted the need to investigate the psychosocial factors impacting frailty in older adults, vital to addressing the health and well-being of the rapidly ageing population in Singapore.

Many studies have tried to explain the mediators and moderators within the pathway of frailty and health outcome $[18-20,21]$ developed a working framework to elaborate the pathway from frailty to its adverse outcomes. In the meantime, [22]. and [18] also elaborated those psychosocial factors in a general matter for health and wellbeing. There are numerous research studies that investigated factors such as: (i) depression being associated with a higher risk of frailty [23-25]. and (ii) living alone lacking in social support and socially isolated had a higher risk of developing frailty [26-28]. Recent findings suggest being older, female, living alone, lacking regular exercise and poor health status are significantly associated with frailty prevalence [29-31] suggested the presence of adverse health outcomes, poor cognition, polypharmacy, sarcopenia, fall rate, living in a private institution or hospital and mortality are related to frailty. Similar findings are supported by [32]. where physiological issues such as poor weight management, disease, and poor psychological health contribute to frailty. Hence, according to Berkman's model [18] and the frailty pathway framework [21]. we examined the existing pathway model on the effects of psychosocial factors on frailty and health outcomes among community-dwelling older adults. In a summary, using Singapore as a case example, the study aimed to investigate the prevalence and related psychosocial factors of frailty in older adults in Singapore and the main research question is: $\mathrm{RQ}$ : What are association between psychosocial factors and frailty and mediate role of frailty between psychosocial factors and Quality of Life (QoL)?

\section{Methodology}

\section{Study design}

This was a cross-sectional study using convenience sampling. Participants underwent a screening phase and a psychosocial profiling phase to gather pre-study data. In the screening phase, upon providing informed consent to take part in the study, participants were issued a questionnaire to gather their basic demographic information and proceeded with the screening session, in which participants were assessed using the frailty scale (Five-item Frail Scale) from [33]. In which the ratings were used to identify the state of frailty in the participants: non-frail (0); pre-frail (1-2); and frail (3-5). Frail participants did not proceed with physical performance assessments. Other self-reported questionnaires include the Katz Activities for Daily Living (ADLs) [34]. and Lawton's Instrumental Activities of Daily Living (IADLs)
[35]. as well as a Fall Efficacy Scale [36], were used. Other biomechanical/physical performance data were collected using the Short Physical Performance Battery (SPPB) [37]. Gait Speed [38]. One-Leg Stand and Grip Strength [33]. In the psychosocial profiling phase, an interviewer-administered questionnaire consisting of validated scales were used to gather data pertaining to participants' psychosocial conditions to measure Depression, Social Support, Quality of Life, and Loneliness (Table 1). Trained personnel (who had undergone training by research staff/ occupational therapist/physiotherapists) conducted the various physical performance assessment components. The study was a single research visit per participant. The entire process took approximately 1 hour per participant.

\section{Measurements}

\section{5-Item frail scale}

The FRAIL scale included 5 components: Fatigue; Resistance; Ambulation; Illness; and Loss of Weight. Frail scale score ranged from 0-5 (i.e., 1 point for each component; 0 =best to $5=$ worst) and represented frail (3-5), pre-frail (1-2), and robust (0) health status.

\section{Katz activities of daily living (ADLs)}

Basic ADLs included seven items (bathing, dressing, eating, transferring bed or chair, walking across a room, getting outside, and using the toilet).

\section{Lawton instrumental activities of daily living (IADLs)}

IADLs included eight items (preparing meals, shopping for groceries, managing money, making phone calls, doing light housework, doing heavy housework, getting to places outside walking distance, and managing medications).

\section{Short physical performance battery (SPPB)}

The SPPB is a summary measure of lower body performance based on three-component tasks: standing balance; chairs stand; and usual walking speed. Each component task was scored as 0-4 (range $0=$ worst to 4 best), and a composite score was computed as the sum of scores on component tasks as 0-12 (range $0=$ worst to $12=$ best $)$.

\section{Gait speed}

Gait speed was assessed in respondents' homes using a standardized 4-meter course with participants instructed to walk at their usual pace. The average walking speed (meters/second) was computed for two trials.

\section{One-leg stand}

For the one-leg stand test, individuals chose their preferred leg to balance on and were required to raise the other foot at least 2 inches above the ground and hold the position for as long as possible up to 30 seconds. 


\section{Grip strength}

Isometric grip strength was assessed using a digital handgrip dynamometer. The mean of the last two maximal effort trials were used in the analysis. The test was performed seated in a chair (without armrests), with feet flat on the floor and the other arm held flat against the side with the elbow at $90^{\circ}$.

\section{Falls efficacy scale}

The Falls Efficacy Scale (FES) measures confidence in performing 10 everyday activities without falling. The response for each FES item ranges from 0 (no confidence) to 10 (complete confidence) and the FES total score ranges from 0-100.

\section{Depression}

The Center for Epidemiologic Studies Depression Scale (CES-D) included 20 items comprising 6 scales reflecting major dimensions of depression [39]. depressed mood; feelings of guilt and worthlessness; feelings of helplessness and hopelessness; psychomotor retardation; loss of appetite; and sleep disturbance. It measured self-reported symptoms associated with depression experienced in the past week. Higher scores represent more depressive symptoms. A cut-off score of 16 indicates high depressive symptoms.

\section{Social support}

The Multidimensional Scale of Perceived Social Support (MSPSS) measured how much support a person feels he or she gets from family, friends, and significant others [40]. The items tended to divide into factor groups relating to the family (FAM), friends (FRI) or significant others (SO). (1=very strongly disagree, $2=$ strongly disagree; $3=$ mildly disagree; $4=$ =neutral; $5=$ mildly agree; $6=$ strongly agree; 7 =very strongly agree).

\section{Quality of life}

The EQ-5D-5L is a standardized instrument to measure generic health status [41]. It is made up of two components: health state description and evaluation. The health state description contained 5 dimensions with 5 response levels (no, slight, moderate, severe, or extreme). The evaluation part measured respondents' overall health status on a visual analog scale (VAS).

\section{Loneliness}

This scale evaluated feelings of loneliness in individuals. An 8-item short form using a 4-point Likert scale ranging from 1 , never, to 4 , always. Minimum and maximum possible scores are 8 and 32, respectively. Higher scores from USL- 8 corresponded to severe loneliness. A cut-off score of 24 was used to classify lonely and not lonely participants based on a previous study [42].

\section{Data Collection}

We conducted a survey study on the target population to identify frailty conditions and psychosocial profiles among older adults in Singapore. A total of 491 community-dwelling older adults were recruited from 17 Senior Activity Centers (SACs)
/ Voluntary Welfare Organizations (VWOs) in Singapore from March to September 2018. The inclusion criteria were: (i) Age >= 65 years (WHO, 2013); (ii) No significant cognitive deficits and can understand and follow instructions; (iii) No significant physical impairments and community ambulant; and (iv) Living in the community and not in a nursing home.

\section{Results \& Analyses}

Calculations were made using the SPSS IBM 25.0 software. A chi-square $\left(\mathrm{c}^{2}\right)$ analysis was performed for intergroup sociodemographic and categorical data. Independent t-tests and analysis of variances were used on continuous data. KruskalWallis $\mathrm{H}$ test was used when assumptions of normality and/or homogeneity was/were violated. Multiple regression was carried out with frailty score and quality of life as dependent variables. In all hypotheses, a significance level of $\alpha=0.05$ was used, and a confidence interval of $95 \%$ was accepted for statistical significance $(\mathrm{p}<0.05)$ at a 2 -tailed level.

\section{Demographics}

A total of 491 eligible older adults took part in the study. The average age of the participants was $(M=74.23, S D=6.25)$ and 127 were males. Socio-demographic findings showed that majority of the study participants were in the age group of 65-74 years $(54.6 \%)$ in three major ethnic groups of Chinese (87.58\%), Malay (8.96\%) and Indian ethnic group (3.46\%). Of the 491 participants, $6.52 \%$ were ascribed to tertiary education, unemployed (never worked and not working) (89.82\%), married (56.1\%) and living with family members $(70.06 \%)$. The variables measuring health status showed that $39.31 \%$ of the study participants had at least two chronic conditions (the combination of hypertension, cardiovascular disease, arthritis, respiratory disease etc.) and $10.79 \%$ had depressive symptoms. About $3.46 \%$ reported feelings of loneliness.

\section{Here are three findings:}

\section{Prevalence of frailty among community-dwelling older adults (65 years and above)}

The prevalence of frailty among community-dwelling older adults aged 65 years old and above in Singapore was at 3.3\%. Approximately $37.1 \%$ and $59.7 \%$ were identified as pre-frail and robust respectively. The socio-demographic information associated with frailty is shown in (Table 1). The prevalence of frailty increased significantly with age, $\mathrm{p}<.001$ among those aged $60-74$ years and 75 years and above, from $31.3 \%$ to $68.8 \%$ respectively. There was a higher prevalence of frailty among females, with marginal significance, $p=.056$. Among ethnic groups, a larger proportion of Chinese was robust as compared to the Malay and Indian ethnic groups. While ethnicity was not significantly associated with frailty status, it is interesting to note that the Indians had a higher proportion of pre-frails at 58.82\% compared to those in robust, $35.29 \%$. Education was significantly different across frailty status, $\mathrm{p}=.036$. Half of the frail participants had no formal education followed by $31.3 \%$ and $18.1 \%$ in primary 
education and secondary education respectively. Employment status was significantly associated with frailty status, $p=.007$, where $100 \%$ of frail participants were not working. There was a higher proportion of married people who were robust $(60.4 \%)$ compared to those who were single, divorced/separated, widowed

Table 1: Frailty Status, Sociodemographic Information and Co-morbidity.

\begin{tabular}{|c|c|c|c|c|}
\hline & $\begin{array}{c}\text { \#Robust } \\
(n=293)\end{array}$ & $\begin{array}{l}\text { \#Prefrail } \\
(n=182)\end{array}$ & $\begin{array}{c}\text { \#Frail } \\
(n=16)\end{array}$ & P Value* \\
\hline Age group (years) $\dagger$ & & & & $<.001$ \\
\hline $65-74$ & $177(60.4)$ & $86(47.3)$ & $5(31.3)$ & \\
\hline $75-84$ & $104(35.5)$ & $75(41.2)$ & $11(68.8)$ & \\
\hline$\geq 85$ & $12(3.5)$ & $21(11.5)$ & $0(0)$ & \\
\hline Sex & & & & 0.056 \\
\hline Male & 87 (29.7) & $36(19.8)$ & $4(25.0)$ & \\
\hline Female & $206(70.3)$ & $146(80.2)$ & $12(75.0)$ & \\
\hline Ethnicity & & & & 0.168 \\
\hline Chinese & $261(89.1)$ & $157(86.3)$ & $12(75.0)$ & \\
\hline Malay & $26(8.9)$ & $15(8.2)$ & $3(18.8)$ & \\
\hline Indian & $6(2.0)$ & $10(5.5)$ & $1(6.3)$ & \\
\hline Education & & & & 0.036 \\
\hline No formal & $70(23.9)$ & $56(30.8)$ & $8(50.0)$ & \\
\hline Primary & $109(37.2)$ & $74(40.7)$ & $5(31.3)$ & \\
\hline Secondary & $88(30.0)$ & $46(25.3)$ & $3(18.8)$ & \\
\hline Tertiary & $26(8.9)$ & $6(3.3)$ & $0(0)$ & \\
\hline Employment status & & & & 0.007 \\
\hline Full-time & $23(7.8)$ & $4(2.2)$ & $0(0)$ & \\
\hline Part-time & $18(6.1)$ & $5(2.7)$ & $0(0)$ & \\
\hline Never worked & $29(9.9)$ & $29(15.9)$ & $0(0)$ & \\
\hline Not working & $223(76.1)$ & $144(79.1)$ & $16(100)$ & \\
\hline Marital status & & & & 0.016 \\
\hline Single, Divorced/separated, widowed & $116(39.6)$ & $95(52.2)$ & $5(31.3)$ & \\
\hline Married & $177(60.4)$ & $87(47.8)$ & $11(68.8)$ & \\
\hline Living arrangement & & & & 0.284 \\
\hline Alone & $80(27.3)$ & $61(33.5)$ & $6(37.5)$ & \\
\hline with family/other people & $213(72.7)$ & $121(66.5)$ & $10(62.5)$ & \\
\hline Comorbidity, mean (SD) & $1.07( \pm 0.94)$ & $1.70( \pm 1.24)$ & $2.81( \pm 1.72)$ & $<.001$ \\
\hline 2 conditions & $83(48.8)$ & $98(77.8)$ & $12(92.3)$ & $<.001$ \\
\hline \multicolumn{5}{|l|}{ Psychosocial condition } \\
\hline Depression & $21(7.2)$ & $25(13.7)$ & $7(43.8)$ & $<.001$ \\
\hline Loneliness & $7(2.4)$ & $8(4.4)$ & $2(12.5)$ & 0.161 \\
\hline
\end{tabular}

"Note: Chi-square test. Values are $\mathrm{n}(\%), \pm$ standard deviation.

(39.4\%). Interestingly, more people were frail in the "Married" (68.8\%) than "those otherwise/not married" categories (31.3\%). A higher proportion of those who lived alone was frail (37.5\%) than prefrail (33.5\%) and robust (27.3\%) (Table 2). 
Table 2: Frailty Status and Functional Measures/Physical Performance Assessments ( $\mathrm{N}=468)$.

\begin{tabular}{|c|c|c|c|}
\hline & $\begin{array}{c}\text { Robust } \\
(\mathbf{n = 2 9 2})\end{array}$ & $\begin{array}{c}\text { Prefrail } \\
(\mathbf{n = 1 7 6})\end{array}$ & P Value* \\
\hline SPPB (0-12 points) & $10.86 \pm 1.82$ & $9.36 \pm 2.55$ & $<.001$ \\
\hline Balance Score & $3.50 \pm 0.89$ & $2.86 \pm 1.31$ & $<.001$ \\
\hline Gait Score & $3.73 \pm 0.62$ & $3.39 \pm 0.83$ & $<.11 \pm 1.18$ \\
\hline Chair Stand Score & $3.63 \pm 0.81$ & $0.88 \pm 0.21$ & $<.001$ \\
\hline Gait Speed (m/s) & $1.00 \pm 0.23$ & $6.04 \pm 8.31$ & $<.001$ \\
\hline One-leg Stand (s) & $10.97 \pm 10.59$ & $17.01 \pm 5.44$ & 0.001 \\
\hline Right Grip Strength (kg) & $18.95 \pm 6.22$ & $17.10 \pm 5.35$ & $<$ \\
\hline Dominant hand Grip Strength (kg) & $19.03 \pm 6.34$ & $<.001$ \\
\hline
\end{tabular}

'Exclude cases listwise. Independent sample t-test were used. Analyses of functional measures only include 'robust' and 'pre-frail' participants as 'frail' participants are omitted as part of the study safety protocol.

Association among frailty, functional status, fall efficacy, physical performance, psychosocial well-being, and healthrelated quality of life

There was a significant difference in IADL functional status, where $\mathrm{p}<.001$ but not ADL functional status. As frailty status progresses from robust to frail, the proportion of older adults with ADL/IAD impairment increases as shown in (Table 3). Significant differences were also seen in Fall efficacy where frail participants had higher mean rank scores compared to prefrail and robust participants (Table 3). Pairwise comparison comparing the threefrailty status be found in (Table 4). Physical performance measures were compared between robust and prefrail participants. Significant differences in physical performance measures between the two groups, $\mathrm{p}<.001$ (Table 2). Robust participants had significantly higher SPPB scores, higher gait speed, better performance in one-leg stand time, and stronger dominant handgrip strength than those in prefrail, $p<.001$. Hence, for the second research question, we conclude that IADL functional status, fall efficacy and Physical performance are significantly associating with frailty. A significantly higher proportion of frail (43.8\%) participants depressed/have high depressive symptoms than prefrail $(13.7 \%)$ and robust $(7.2 \%)$ participants, where $\mathrm{p}<.001$. However, there was no significant difference in the proportion of participants who were lonely across frailty status. Significant differences were observed in loneliness and depression scores across frailty status, $\mathrm{p}<.001$ (Table 2). Loneliness score was significantly higher in both prefrail and frail participants than robust participants, where $p=.001$. Robust participants had a significantly lower score depression score than prefrail and frail participants respectively, $\mathrm{p}<.001$. Kruskal-Wallis $\mathrm{H}$ tests showed that there was a significant difference in overall social support between the different frailty status, $\chi^{2}(2)=18.76, p<.001$. Multiple Pairwise comparisons showed that robust participants were significantly higher than frail participants (Mean Rank = 166.66), $\mathrm{p}=.017$, and robust participants (Mean Rank =267.55) were significantly higher than pre-frail participants (Mean Rank = 218.28), $p=.001$. Social support from the subscales from friends
(FR), family (FM), and a significant other (SO) was also found to be significant. Multiple pairwise comparisons results can be found in (Table 4). We measured frailty with health-related quality of life. Self-reported health status was significantly lower in frail, followed by prefrail, and robust participants (Table 3). Frailty was significantly associated with having problems in all domains of health dimensions where robust participants had the least problems except in anxiety/depression. (Figure 1) shows the percentage/proportion of frail, prefrail, and robust participants who had problems with all five health domains (EQ-5D). The top two domains frail participants had problems with were pain/ discomfort (81.3\%) and mobility (50\%). Self-evaluated overall health status was significantly lower in frail participants followed by prefrail and robust (Table 3). Findings showed that factors affecting psychosocial well-being and health-related quality of life are significantly associated with frailty.

\section{Validation of the mediation effect of frailty through pathway analyses}

Based on the existing pathway models [19-21]. We selected the existing model linking psychosocial factors $(X)$ to quality of life $(\mathrm{Y})$, which is conditional if the indirect effect of $\mathrm{X}$ on $\mathrm{Y}$ through $\mathrm{M}$ (a mediator variable) depends on $\mathrm{W}$ (a moderator variable) (Figure 2). To validate the mediation effect of frailty among psychosocial factors and quality of life, two regression models to obtain the path coefficients to determine whether independent variables like depression, loneliness, social support, and frailty scores would significantly predict the quality of life, the outcome dependent variable. Multiple regression was run to predict the quality of life from loneliness, depression, social support, and frail scores. The multiple regression model statistically significantly predicted quality of life, $\mathrm{F}(4,485)=25.891, \mathrm{p}<.001$, adj. $\mathrm{R} 2=.176$. All variables added statistically significantly to the prediction, $\mathrm{p}<$ .05 except for social support, $\mathrm{p}=.558$ and loneliness, $\mathrm{p}=.066$. Regression coefficients and standard errors can be found in (Table 5). (Figure 3) shows the frailty mediational hypothesis model from psychosocial factors to quality of life. The red and black lines represent the significant and non-significant relationships. 
A cumulative odds ordinal logistic regression with proportional odds was run to determine whether depression, loneliness and social support affect the frail scores. A decrease in social support was associated with an increase in the odds of higher frail scores, with an odds ratio of .832 (95\% CI, .711 to .972), Wald $\chi^{2}(1)=$ $5.369, \mathrm{p}=.020$. An increase in depression scores was associated with an increase in the odds of higher frail scores, with an odds ratio of 1.071 ( $95 \% \mathrm{CI}, 1.037$ to 1.105$)$, Wald $\chi^{2}(1)=17.814, \mathrm{p}$
$<.0005$. A decrease in loneliness was not associated with an increase in the odds of higher frail scores, with an odds ratio of $.982(95 \%$ CI, .930 to 1.036$)$, Wald $\chi^{2}(1)=.444, p=.505$. These results supported the existing mediational hypothesis model, which revealed that the indirect effect of depression, loneliness, and social support on quality of life was mediated by frailty. Depression had both direct and indirect impacts on quality of life.

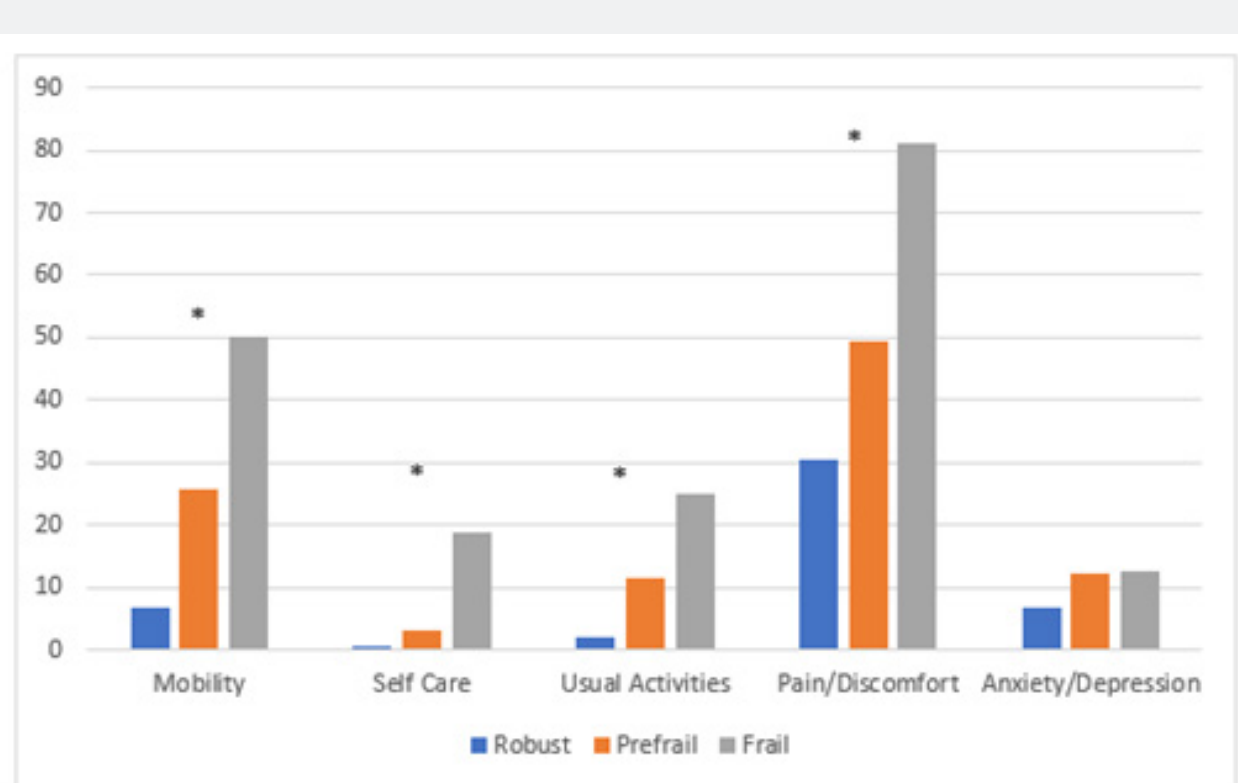

Figure 1: Frailty and Quality of Life (EQ-5D) * Represents the statistical significant difference.

Table 3: Frailty Status, Functional Impairment/disability, falls-efficacy, and psychosocial well-being/measures.

\begin{tabular}{|c|c|c|c|c|}
\hline & $\begin{array}{c}\text { \#Robust } \\
(n=293)\end{array}$ & $\begin{array}{l}\# \text { Prefrail } \\
(n=182)\end{array}$ & $\begin{array}{c}\text { \#Frail } \\
(n=16)\end{array}$ & P Value* \\
\hline ADL & & & & 0.142 \\
\hline No ADL impairment/disability & $275(93.9)$ & $167(91.8)$ & $13(81.3)$ & \\
\hline$\geq 1$ ADL impairment/disability & $18(6.1)$ & $15(8.2)$ & $3(18.8)$ & \\
\hline IADL & & & & $<.001$ \\
\hline No IADL impairment/disability & $250(85.3)$ & $129(70.9)$ & $8(50.0)$ & \\
\hline$\geq 1$ IADL impairment/disability & $43(14.7)$ & $53(29.1)$ & $8(50.0)$ & \\
\hline \multicolumn{5}{|l|}{ Other self-reported measurements } \\
\hline Falls Efficacy§, Mean Rank & 209.26 & 295.93 & 350.78 & $<.001$ \\
\hline Depression§ Mean Rank & 216.13 & 283.75 & 363.56 & $<.001$ \\
\hline Loneliness§ Mean Rank & 225.08 & 274.73 & 302.34 & $<.001$ \\
\hline Social Support, mean§, Mean Rank & 267.55 & 218.28 & 166.66 & $<.001$ \\
\hline SO=§, Mean Rank & 261.66 & 224.1 & 208.44 & 0.01 \\
\hline $\mathrm{FM}=\S$ Mean Rank & 261.65 & 227.96 & 164.66 & 0.002 \\
\hline $\mathrm{FR}=\S$, Mean Rank & 265.6 & 218.61 & 198.59 & 0.001 \\
\hline Mean subjective QOL+ & $85.09 \pm 12.61$ & $79.88 \pm 16.54$ & $71.44 \pm 19.14$ & $<.001$ \\
\hline
\end{tabular}

Values in $\mathrm{n}(\%), \pm$ standard deviation. + Based on EQ-5D-5L measures of subjective health. = Social Support subscales SO represents Social support from a 'Significant Other', FM represents social support form 'Family', and FR represents social support from 'Friends'. § Kruskal-Wallis Test was used as assumption of normality is violated, values in Mean Rank. For continuous data, ANOVA or Kruskal- Wallis $\mathrm{H}$ test was used. 


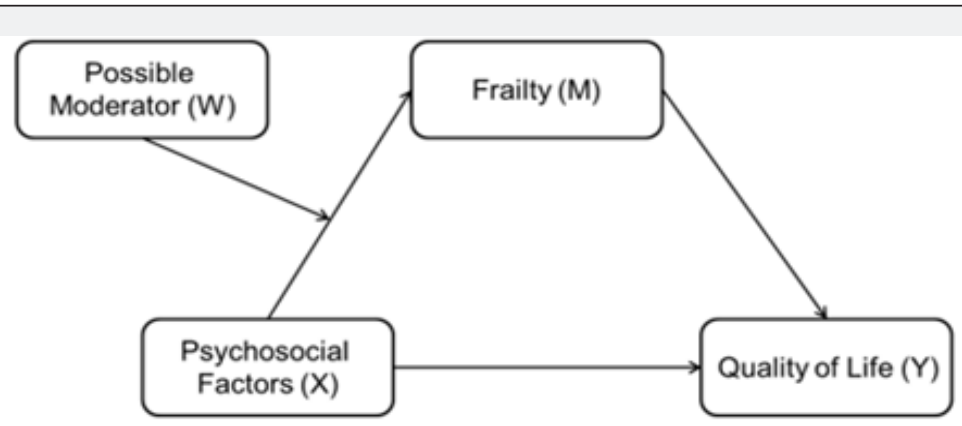

Figure 2: Models of the relationships between Psychosocial Factors, Frailty and Quality of Life.

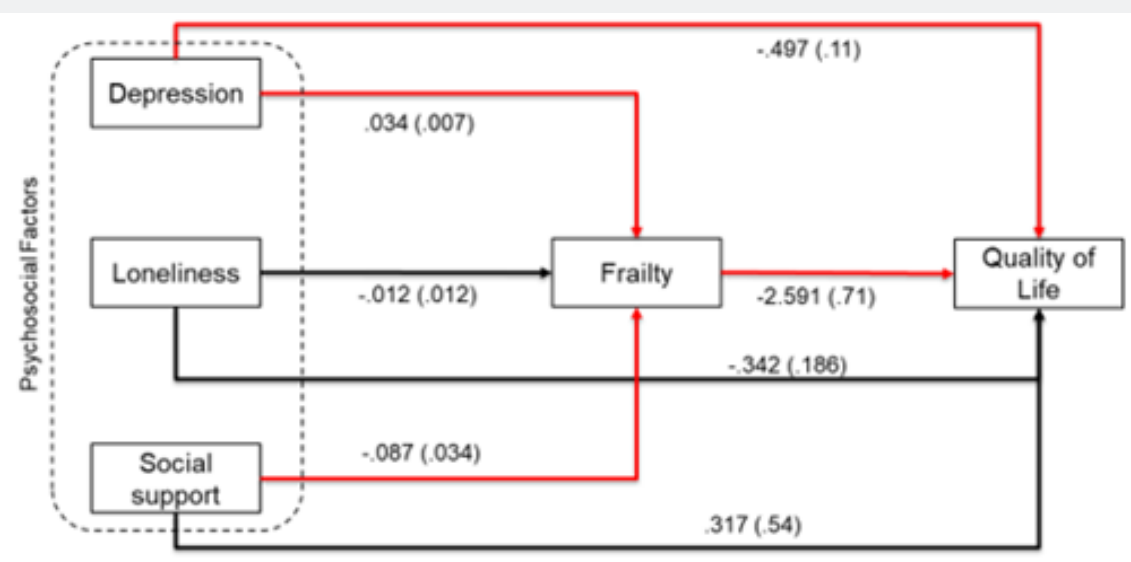

Figure 3: Mediation analysis model scores in unstandardized regression coefficient.

Table 4: Kruskal Wallis Test and Multiple Comparisons on Fall Efficacy, Depression, Loneliness, Social Support.

\begin{tabular}{|c|c|c|c|c|c|c|c|}
\hline \multicolumn{3}{|c|}{ Other Self-Reported Measurements } & \multicolumn{2}{|c|}{ Kruskal-Wallis Test } & \multicolumn{3}{|c|}{ Pairwise Comparison } \\
\hline & $\mathbf{N}$ & $\mathbf{H}$ & Df & $P$ value & & $P$ value & Test statistic \\
\hline \multirow[t]{3}{*}{ Falls Efficacy } & 491 & 60.47 & 2 & $<.001^{*}$ & Robust-Prefrail & $<.001^{* *}$ & -86.67 \\
\hline & & & & & Robust-Frail & $<.001^{* *}$ & -141.52 \\
\hline & & & & & Prefrail-Frail & 0.318 & -54.85 \\
\hline \multirow[t]{3}{*}{ Depression } & 491 & 37.05 & 2 & $<.001^{*}$ & Robust-Prefrail & $<.001^{* *}$ & -67.62 \\
\hline & & & & & Robust-Frail & $<.001^{* *}$ & -147.431 \\
\hline & & & & & Prefrail-Frail & 0.092 & -79.82 \\
\hline \multirow[t]{3}{*}{ Loneliness } & 491 & 16.74 & 2 & $<.001^{*}$ & Robust-Prefrail & $.001^{* *}$ & -49.65 \\
\hline & & & & & Robust-Frail & 0.096 & -77.265 \\
\hline & & & & & Prefrail-Frail & 1 & -27.62 \\
\hline \multirow[t]{3}{*}{ Social Support } & 491 & 18.76 & 2 & $<.001^{*}$ & Frail-Prefrail & 0.487 & 51.621 \\
\hline & & & & & Frail-Robust & $.017^{* *}$ & 100.9 \\
\hline & & & & & Prefrail-Robust & $.001^{* *}$ & 49.28 \\
\hline \multirow[t]{3}{*}{ so } & 491 & 9.25 & 2 & $.010^{*}$ & Frail-Prefrail & 1 & 15.66 \\
\hline & & & & & Frail-Robust & 0.417 & 53.22 \\
\hline & & & & & Prefrail-Robust & $.014^{* *}$ & 37.56 \\
\hline
\end{tabular}


Juniper Online Journal of Public Health

\begin{tabular}{|c|c|c|c|c|c|c|c|}
\hline FM & 491 & 12.33 & 2 & $.002^{*}$ & Frail-Prefrail & 0.239 & 63.3 \\
\hline & & & & & Frail-Robust & $.019 * *$ & 96.99 \\
\hline & & & & & Prefrail-Robust & $.030^{* *}$ & 33.69 \\
\hline FR & 491 & 14.27 & 2 & $.001^{*}$ & Robust-Prefrail & 1 & 20.02 \\
\hline & & & & & Robust-Frail & 0.194 & $.001^{* *}$ \\
\hline
\end{tabular}

*: Difference between the three-frailty status was statistically significant at $p=.05$ )

SO represents Social Support from 'Significant Other', FM represents Social Support from 'Family', FR represents Social Support from 'Friends'

**: Significance values have been adjusted by the Bonferroni correction for multiple tests.

$\mathrm{N}$ : Total number of respondents, $\mathrm{H}$ : Kruskal-Wallis test statistic, df: degree of freedom.

Table 5: Multiple regression results for Quality of Life (QOL).

\begin{tabular}{|c|c|c|c|c|c|c|c|}
\hline QOL & B & \multicolumn{2}{|c|}{$\mathbf{9 5 \%}$ CI For B } & SE B & $\boldsymbol{\beta}$ & $\mathbf{R}^{2}$ & $\mathbf{\Delta R}^{\mathbf{2}}$ \\
\hline & & $\mathbf{L L}$ & $\mathbf{U L}$ & & & \\
\hline Model & $90.476^{* * *}$ & 81.87 & 99.08 & 4.38 & & 0.176 \\
\hline Loneliness & -0.342 & -0.71 & 0.02 & 0.187 & -0.11 & \\
\hline Depression & -0.497 & -0.72 & -0.28 & 0.11 & $-.25^{* * *}$ & \\
\hline Social Support & 0.317 & -0.75 & 1.38 & 0.54 & 0.03 & \\
\hline FRAIL score & -2.591 & -0.399 & -1.19 & 0.71 & $-.16^{* * *}$ & \\
\hline
\end{tabular}

Note: $\mathrm{QOL}=$ Quality of Life. Model = "Enter" method in SPSS Statistics; $\mathrm{B}=$ unstandardized regression coefficient; $\mathrm{Cl}=$ confidence interval; $\mathrm{LL}=$ lower limit; $\mathrm{UL}=$ upper limit; $\mathrm{SE} B=$ standard error of the coefficient; $\beta=$ standardized coefficient; $\mathrm{R}^{2}=$ coefficient of determination; $\Delta \mathrm{R}^{2}=$ adjusted $R^{2} .{ }^{*} p<.05 .{ }^{* *}<.01 .{ }^{* * *} p<.001$.

\section{Discussion}

This study investigated the prevalence of frailty among community older adults (65 years and above) in Singapore. Based on our findings, the prevalence of frailty (3.3\%) is similar to earlier study conducted from 2010 to 2013 in Singapore [16,17]. Compared to other countries where the prevalence of frailty ranged from $4 \%$ to $44 \%$ [8]. The prevalence of frailty among the older adults in Singapore is at the lower end of the spectrum at $3.3 \%$, and early interventions at personal, community and societal levels are needed before the problem becomes serious. Results also revealed that the prevalence of frail and pre-frail states increased with age, which is in line with the general literature [8]. The study also showed that approximately $31.3 \%$ of those aged 60-75 years were frail, showing the importance of assessing frailty even among those aged $<60$ years. We observed ethnic differences in pre-frailty and frailty, concurring with findings from a study by [16]. Singapore primarily has three main ethnic groups, namely, Chinese (74.3\%); Malays (13.5\%); Indians (9.0\%) [1]. Chinese older adults were found to have a lower prevalence of both frailty $(2.79 \%)$ and pre-frailty (36.5\%) at the bivariate level, compared to Indian older adults who were found to have almost twice the odds for frailty (5.88\%) and pre-frailty (58.82\%) (Table 1). However, Malay older adults have a prevalence of frailty $(6.82 \%)$ and prefrailty (34.09\%). [43] and [44] pointed out that frailty is more prevalent in ethnic minorities, as cultural factors, and lifestyle choices in turn lead to variations in health habits and access to resources. Older adults may benefit from the multicultural society and environment in Singapore, hence the prevalent difference between the ethnic group is not significant. More longitudinal studies to understand relationships between frailty and social support are pertinent in formulating customized intervention programs for the different ethnic groups in Singapore.

While many studies have already done on predictors of physical frailty in older people, less is known on psychosocial factors for whom and how they exert their effects. Based on the Bergman conceptual framework and possible model identified recently $[19,21]$. The adopted conditional process model is the indirect effect of depression, loneliness, and social support on Quality of Life through frailty that is moderated (Figure 2). Observing associations between quality of life and conditions measured concurrently does not necessarily permit understanding of the direction of effect. Rather, these associations describe how Quality of Life levels vary with a broad set of psychological conditions in older people. Based on our findings, frailty is one mediator among the psychosocial factors to Quality of Life, while age, gender, ethnicity, education, employment, and marital status are possible moderators on pathways from psychological conditions to Quality of Life.

\section{Conclusion}

As a summary, establishing frailty prevalence and its related psychosocial factors is undoubtedly important for both clinical practice and the national healthcare system. According to our study results, the prevalence of frailty and pre-frailty are $3.3 \%$ 
and $37.1 \%$; the results also investigated the association between psychosocial factors and frailty and mediate role of frailty between psychosocial factors and Quality of Life. The study has several clinical, research, and policy implications. Though sampling participants and periods are limited, the psychosocial factors impacting frailty may be underestimated. Preliminary findings have shown the factors (social support, depression, loneliness and fall efficacy) associated with frailty and pre-frailty which require a need for greater collaboration between health professionals, social services and researchers concerned with the health and well-being of older adults in the community. This study's findings confirmed that depression, loneliness, and social support have direct and indirect impacts on quality of life. Hence, a good social functioning with the meaningful networked community is important as it nudges healthy ageing and reduced vulnerability [45]. And loneliness.

\section{Acknowledgements}

We would like to thank the research team from Centre for Healthy and Sustainable Cities (CHESS), Wee Kim Wee School of Communication and Information for data collection, clinicians from Tan Tock Seng Hospital and Singapore General Hospital for their inputs towards the research design, and Rehabilitation Research Institute of Singapore (RRIS) for providing training of the physical performance assessments. We would also thank intern Chua Hui Min for preparing related references.

\section{Declaration of Conflicting Interests}

The author(s) declared no potential conflicts of interest with respect to the research, authorship, and/or publication of this article.

\section{References}

1. (2020) Department of Statistics Population Trends.

2. Yap MT, Gee C (2014) Population Outcomes Singapore 2050.

3. Walston J (2006) Research agenda for frailty in older adults toward a better understanding of physiology and etiology. J Am Geriatr Soc 54.

4. Song X, Mitnitski A, Rockwood K (2010) Prevalence and 10-Year outcomes of frailty in older adults in relation to deficit accumulation. J Am Geriatr Soc 58.

5. Gitlin LN, Winter L, Stanley IH (2017) Compensatory Strategies Prevalence of Use and Relationship to Physical Function and WellBeing. J Appl Gerontol 36: 647-666.

6. Lynch M, Estes CL (2005) Chronic Care Initiatives for the Elderly Can They Bridge the Gerontology-Medicine Gap. J Appl Gerontol 24: 108124.

7. Stillman GR, Stillman AN, Beecher MS (2021) Frailty Is Associated with Early Hospital Readmission in Older Medical Patients. J Appl Gerontol 40: 38-46.

8. Collard RM, Boter H, Schoevers RA, Voshaar RCO (2012) Prevalence of frailty in community-dwelling older persons A systematic review. Journal of the American Geriatrics Society 60.

9. Ofori Asenso R (2019) Global Incidence of Frailty and Prefrailty Among Community-Dwelling Older Adults A Systematic Review and Metaanalysis. JAMA Netw open 2.
10. Clegg A, Young J, Iliffe S, Rikkert MO, Rockwood K (2013) Frailty in elderly people. Lancet 381 .

11. Ensrud KE (2007) Frailty and risk of falls fracture and mortality in older women the study of osteoporotic fractures. J Gerontol A Biol Sci Med Sci 62: 744-751.

12. Al Snih S (2009) Frailty and incidence of activities of daily living disability among older Mexican Americans. J Rehabil Med 41: 892-897.

13. Ge L, Yap CW, Heng BH (2019) Prevalence of frailty and its association with depressive symptoms among older adults in Singapore. Aging Ment Health 23: 319-324.

14. Cheong CY (2020) Risk Factors of Progression to Frailty Findings from the Singapore Longitudinal Ageing Study. J Nutr Health Aging 24: 98106.

15. Lim WS, Wong CH, Ding YY, Rockwood K (2019) Translating the Science of Frailty in Singapore Results from the National Frailty Consensus Discussion. Ann Acad Med Singapore 48: 25-31.

16. Vaingankar JA (2017) Prevalence of frailty and its association with sociodemographic and clinical characteristics and resource utilization in a population of Singaporean older adults. Epidemiol Clin Pract Heal 17: $1444-1454$.

17. Ng TP, Feng L, Nyunt MSZ, Larbi A, Yap KB (2014) Frailty in older persons multisystem risk factors and the Frailty Risk Index (FRI). J Am Med Dir Assoc 15: 635-642.

18. Berkman LF, Krishna A (2014) Social network epidemiology.

19. Mehrabi F, Béland F (2020) Effects of social isolation loneliness and frailty on health outcomes and their possible mediators and moderators in community dwelling older adults A scoping review. Arch Gerontol Geriatr 90: 104119.

20. Ding YY, Kuha J, Murphy M (2017) Pathways from physical frailty to activity limitation in older people Identifying moderators and mediators in the English Longitudinal Study of Ageing. Exp Gerontol 98: $169-176$.

21. Bergman H (2004) Developing a working framework for understanding frailty. Gérontologie et société 15-29.

22. Umberson D, Crosnoe R, Reczek C (2010) Social Relationships and Health Behavior Across Life Course. Annu Rev Sociol 36: 139-157.

23. Ottenbacher KJ (2009) Mexican Americans and frailty findings from the Hispanic established populations epidemiologic studies of the elderly. Am J Public Health 99: 673-679.

24. Lakey SL (2012) Antidepressant use depressive symptoms and incident frailty in women aged 65 and older from the Women's Health Initiative Observational Study. J Am Geriatr Soc 60: 854-861.

25. Park Lee E, Fredman L, Hochberg M, Faulkner K (2009) Positive affect and incidence of frailty in elderly women caregivers and noncaregivers results of Caregiver Study of Osteoporotic Fractures. J Am Geriatr Soc 57: 627-633.

26. Etman A, Burdorf A, Vander Cammen TJM, Mackenbach JP, Van Lenthe FJ (2012) Socio-demographic determinants of worsening in frailty among community-dwelling older people in 11 European countries. J Epidemiol Community Health 66: 1116-1121.

27. Ntanasi E (2020) Prevalence and Risk Factors of Frailty in a CommunityDwelling Population The HELIAD Study. J Aging Health 32: 14-24.

28. Peek MK, Howrey BT, Ternent RS, Ray LA, Ottenbacher KJ (2012) Social support stressors and frailty among older Mexican American adults. J Gerontol B Psychol Sci Soc Sci 67: 755-764.

29. Liu W (2020) Physical frailty and its associated factors among elderly nursing home residents in China. BMC Geriatr 20. 


\section{Juniper Online Journal of Public Health}

30. He B (2019) Prevalence and Risk Factors for Frailty Among Community Dwelling Older People in China a Systematic Review and Meta Analysis. Journal of Nutrition Health and Aging 23.

31. Fhon JRS (2018) Factors associated with frailty in older adults A longitudinal study. Rev Saude Publica 52.

32. Espinoza SE, Fried LP (2007) Risk Factors for Frailty in the Older Adult Credit Designation Statement. Clin Geriatr 15.

33. Morley JE, Malmstrom TK, Miller DK (2012) A simple frailty questionnaire (FRAIL) predicts outcomes in middle aged African Americans. J Nutr Health Aging 16: 601-608.

34. Katz S, Downs TD, Cash HR, Grotz RC (1970) Progress in development of the index of ADL. Gerontologist 10: 20-30.

35. Lawton MP, Brody EM (1969) Assessment of Older People Self Maintaining and Instrumental Activities of Daily Living. Gerontologist 9: 179-186.

36. Tinetti ME, Richman D, Powell L (1990) Falls efficacy as a measure of fear of falling. J Gerontol 45: 239-243.

37. Guralnik JM (1994) A short physical performance battery assessing lower extremity function association with self-reported disability and prediction of mortality and nursing home admission. J Gerontol 49: 85-94.
38. Abellanvan Kan G (2009) Gait speed at usual pace as a predictor of adverse outcomes in community-dwelling older people an International Academy on Nutrition and Aging (IANA) Task Force. J Nutr Health Aging 13: 881-889.

39. Radloff LS (1977) The CES D Scale a Self-Report Depression Scale for Research in the General Population. Appl Psychol Meas 1: 385-401.

40. Zimet GD, Dahlem NW, ZimetSG, Farley GK (1988) The Multidimensional Scale of Perceived Social Support. J Pers Assess 52: 30-41.

41. Herdman M (2011) Development and preliminary testing of the new five-level version of EQ-5D (EQ-5D-5L). Qual life Res 20: 1727-1736.

42. Wang J (2020) Epidemiology of loneliness in a cohort of UK mental health community crisis service users. Soc Psychiatry Psychiatr Epidemiol 55.

43. Xue QL (2011) The frailty syndrome definition and natural history. Clin Geriatr Med 27: 1-15.

44. Bandeen Roche K (2015) Frailty in Older Adults a Nationally Representative Profile in the United States. J Gerontol A Biol Sci Med Sci 70: 1427-1434.

45. Tallman PS (2016) The Index of Vulnerability An anthropological method linking social-ecological systems to mental and physical health outcomes. Soc Sci Med 162: 68-78.

Your next submission with Juniper Publishers will reach you the below assets

Commons Attribution 4.0 License

DOI: $10.19080 / J O J P H .2021 .06 .555687$ 